\title{
Cell cycle control of DNA joint molecule resolution
}

\author{
Philipp Wild and Joao Matos ${ }^{1}$
}

Institute of Biochemistry, HPM D6.5 - ETH Zürich, Otto-Stern-Weg 3, 8093 Zürich, Switzerland.

${ }^{1}$ Corresponding author: tel +(41) 446336115 e-mail: joao.matos@bc.biol.ethz.ch

Key words: Genome stability, chromosome segregation, structure-selective endonucleases, Mus81, Yen1/GEN1, CDK, Cdc5, Cdc14, APC/C 


\section{SUMMARY}

The establishment of stable interactions between chromosomes underpins vital cellular processes such as recombinational DNA repair and bipolar chromosome segregation. On the other hand, timely disengagement of persistent connections is necessary to assure efficient partitioning of the replicated genome prior to cell division. Whereas great progress has been made in defining how cohesinmediated chromosomal interactions are disengaged as cells prepare to undergo chromosome segregation, little is known about the metabolism of DNA joint molecules (JMs), generated during the repair of chromosomal lesions. Recent work on Mus81 and Yen1/GEN1, two conserved structure-selective endonucleases, revealed unforeseen links between JM-processing and cell cycle progression. Cell cycle kinases and phosphatases control Mus81 and Yen1/GEN1 to restrain deleterious JM-processing during S-phase, while safeguarding chromosome segregation during mitosis. 


\section{Introduction:}

As the genome is recurrently exposed to endogenous and exogenous stresses, accurate inheritance of genetic information requires continuous repair of DNA lesions [1,2]. Homologous recombination $(\mathrm{HR})$, the error-free DNA double-strand break (DSB) repair pathway, utilizes the intact sister chromatid (or on rare instances the homologous chromosome) as a template to synthesize the missing DNA sequence and re-join the broken ends $[3,4]$. This process, however, entails the formation of stable DNA connections between chromosomal arms, which need to be disengaged prior to cell division. Therefore, while contributing to genome stability, recombinational DNA repair promotes formation of dangerous intermediates, which require especial attention from cells.

To separate DNA joint molecules (JMs) that form during HR, proliferating cells (i.e. cells that give rise to progeny through mitotic division) are endowed with various JM-processing enzymes. Anti-recombinogenic helicases such as Mph1/FANCM, Srs2 and RTEL1 disengage the majority of early JMs resulting in the repair of DSBs without the reciprocal exchange of flanking DNA sequences (non-crossover) [5-7] (Figure 1A and B). If left unprocessed, early JM intermediates mature into four-way junctions - also known as Holliday junctions (HJs) - in which sister chromatids (or homologous chromosomes) become covalently linked $[8,9]$. Due to their stability in connecting the two DNA duplexes, HJs are arguably the most dangerous of all recombination intermediates (Figure 1A-C). Interestingly, HR-mediated DSB repair is not the only source of HJs. Four-way DNA junctions that resemble canonical HJs can 
also arise, for instance, upon replication stress and replication fork reversal [10].

To ensure robust processing of late JMs, eukaryotic cells rely on at least three genetically and biochemically distinct pathways: the STR/BTR complex (yeast

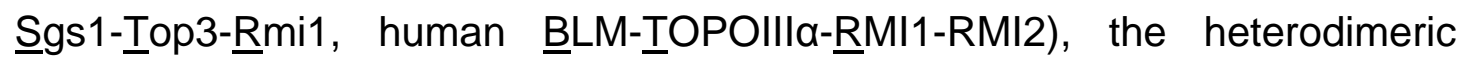
structure-selective endonuclease Mus81 (Mus81-Mms4 in budding yeast, Mus81-Eme1 in fission yeast, MUS81-EME1 and MUS81-EME2 in human cells) and the $\mathrm{HJ}$ resolvase Yen1/GEN1 [11-15]. STR/BTR migrates and decatenates double Holliday junctions ( $\mathrm{dHJs}$ ) by a mechanism termed "dissolution". Mus81 and Yen1/GEN1 cut individual HJs through nucleolytic "resolution" (Figure 1D) [16]. It is important to point out that the functions of Mus81 and Yen1/GEN1 are not limited to HJ processing. Both nucleases are thought to cleave, for example, HJ precursors, such as nicked HJs [12,17-19]. Furthermore, at least in mammalian cells, HJ incision by MUS81 requires prenicking of the opposite strand by the structure-selective endonuclease SLX1SLX4 [20-25].

Besides covalently connecting recombining DNA duplexes, HJs entail a second feature that cannot be underestimated by cells: their processing can lead to the incidence of reciprocal genetic exchanges (crossovers). Hence, if the template used for repair is the homologous chromosome, instead of the sister chromatid, loss of heterozygosity ( $\mathrm{LOH}$ ) can ensue (Figure 1A). To suppress crossovers (COs), and the potential for $\mathrm{LOH}$, proliferating cells dissolve most dHJs using the STR/BTR pathway, which leads to formation of non-crossover (NCO) recombinants, exclusively [5,11]. Mus81 and 
Yen1/GEN1, which resolve HJs to generate both COs and NCOs, also contribute to JM processing. However, both enzymes appear to function as a backup to STR/BTR, or whenever JMs contain single HJs, which require nucleolytic resolution [17,26-29]. It is important to mention that HR drives genetic exchange and the creation of new parental alleles in germ cells undergoing meiosis. To this end, cells modify JM processing significantly [30]. For example, during meiosis, the mismatch repair factors Mlh1, Mlh3 and Exo1 act in a fourth pathway of HJ processing, which channels a substantial fraction of JMs to strictly generate COs [31].

So how do proliferating cells configure STR/BTR, Mus81 and Yen1/GEN1 to ensure efficient DNA repair, while minimizing the occurrence of COs? In recent years, accumulating evidence from different organisms revealed that pathway usage is tightly controlled by cell cycle kinases and phosphatases, and is thus coordinated with progression through the cell cycle. This review focuses on how budding yeast cells wire Mus81-Mms4 and Yen1 activation to cell cycle progression, which precludes toxic processing of recombination intermediates during S-phase and safeguards resolution of persistent DNA JMs during mitosis.

\section{Cell cycle regulation of DNA joint molecule processing}

Budding yeast cells exhibit temporal separation in the formation of $\mathrm{CO}$ and NCO recombinants upon DSB repair [5]. In proliferating cells, STR dissolves most $\mathrm{dHJ}$ at early stages of the cell cycle, to generate NCO recombinants. In STR mutants, however, JMs persist until later stages of the cell cycle when 
they are processed to generate both COs and NCOs $[5,26]$. Since Mus81 and Yen1 functionally overlap with STR and can process HJs to generate a mixture of COs and NCOs $[28,29,32]$, a model was put forward in which both nucleases resolve JMs that escape STR-mediated HJ dissolution. These would occasionally include dHJs, but also single HJs and nicked HJs (Figure $1 A$ and $B)$.

How cells establish such temporal separation in pathway usage has been a subject of intense research. Consequently, the last four years have brought us considerable progress in the delineation of the cellular mechanism used to provide STR the leading role in JM processing. While it remains unclear whether the function of STR is cell cycle-regulated, evidence for stagespecific activation of Mus81 and Yen1/GEN1 has emerged from different organisms, including $S$. cerevisiae, S. pombe and human cells in culture [20,33-39]. As the mechanistic details of Mus81 and Yen1/GEN1 regulation have been reviewed elsewhere [40,41], we will only discuss the key points here.

\section{Control of Mus81 and Yen1 nucleases by cell cycle kinases and phosphatases}

Several studies have provided complementary insight into the elegant modes of regulation of the Mus81 and Yen1 nucleases [34,37,39,42]. Both enzymes are regulated by cell cycle stage-specific phosphorylation events that impose temporal control on their actions and lead to their sequential activation. Mus81-Mms4 activity is regulated by cell cycle stage-specific phosphorylation 
events that render the nuclease most active at the $\mathrm{G}_{2} / \mathrm{M}$ transition [42] (Figure 2). This cyclic pattern of activation is reminiscent of the oscillating activities of cyclin-dependent kinases (CDKs), which drive orderly progression through the cell cycle by changing the properties of a plethora of substrates [43]. Indeed, M-phase CDK (Cdc28 in budding yeast) collaborates with a second cell cycle kinase, Polo-like kinase Cdc5, in modifying Mus81-Mms4 at the onset of mitosis $[34,36,37,39,42,44]$. More specifically, multiple phosphorylation events on the non-catalytic subunit Mms4 trigger hyperactivation of the Mus81-Mms4 nuclease complex. Precisely how phosphorylation enhances Mus81 activity and function remains to be elucidated.

Yen1 function is similarly under the control of cell cycle stage-specific modifications, which impinge on its catalytic activity as well as on its subcellular localization (Figure 2A). However, contrary to Mus81, extensive phosphorylation of Yen1 by S-phase CDKs down-regulates its activity and, alongside, directs Yen1 away from its nuclear substrates, into the cytoplasm. At the onset of anaphase, the cell cycle phosphatase Cdc14 promotes the reverse reaction allowing Yen1 to re-shuttle into the nucleus (Figure 2A) $[33,38,42]$. Mechanistically, on the one hand, phosphorylation lowers the affinity of Yen1 to its negatively charged DNA substrate, on the other hand, renders its nuclear localization signal nonfunctional, thus impairing its nuclear accumulation $[33,38,45]$.

Collectively, the work described above began to elucidate how cells adjust the usage of JM-processing enzymes. To prioritize STR-mediated dissolution, cells temporally and spatially restrain Mus81-Mms4 and Yen1 functions. This generates a wave of JM dissolution during S-phase that is followed by two 
consecutive waves of JM resolution during mitosis, reducing enzymesubstrate competition. Such pattern of regulation reduces the occurrence of mitotic $\mathrm{COs}$, limiting the potential for $\mathrm{LOH}$, while also ensuring the resolution of persistent JMs, which could interfere with chromosome segregation and lead to aneuploidy.

As predicted from the model described in Figure 1, mutations that cause premature Mus81-Mms4 or Yen1 activation induce pathway competition and trigger a significant increase in mitotic $\mathrm{CO}$ and $\mathrm{LOH}[33,34,38,39]$. However, this is not the only phenotype associated with untimely nuclease activation. Interestingly, premature activation of Mus81-Mms4 and Yen1 during S-phase appears to be deleterious to DNA replication and repair $[33,36,39]$. Thus, it is possible that the tight control of Mus81-Mms4 and Yen1 function also relates to their limited substrate specificity. Since both nucleases are proficient in cleaving model replication forks and other branched DNA intermediates, it is likely that the restraint of their activities during S-phase prevents the toxic processing of vital DNA replication and repair intermediates.

\section{Wiring DNA joint molecule resolution to cell cycle progression: parallels between Mus81/Yen1 and $A P C / C^{C d c 20 / C d h 1}$ regulation}

One remarkable feature of the consecutive but temporally separable waves of JM processing by Mus81-Mms4 and Yen1 is that they peak at metaphase and anaphase, respectively. As such, both nucleases complement each other in resolving JMs at the onset and throughout all stages of mitosis (Figure 2) [42]. As pointed out earlier, this is achieved through a common regulator, CDK, which activates Mus81 and inactivates Yen1, creating two interlinked waves 
of JM resolution that are absent during S-phase while covering all stages of mitosis, as well as G1 (Figure 2). This strategy used to reduce JM resolution during S-phase while enhancing it at all other stages of the cell cycle may appear complex at a first glance. However, activation of Mus81-Mms4 and Yen1 shares remarkable similarities to the regulation of one of the most prominent CDK substrates required for the segregation of chromosomes, the anaphase-promoting complex/cyclosome (APC/C) [46].

The ubiquitin ligase $\mathrm{APC} / \mathrm{C}$ is indispensable for the rapid and irreversible metaphase to anaphase transition, as well as for mitotic exit $[46,47]$. The metaphase-anaphase transition is achieved by ubiquitin-dependent proteasomal degradation of securin, which inhibits the protease separase that upon cleavage of cohesin ultimately triggers sister chromatid segregation. Mitotic exit relies on cyclin B degradation, which results in M-phase CDK inactivation [48-51]. Since the APC/C plays a crucial role in genome stability by facilitating accurate chromosome segregation, it comes as no surprise that its activity is subject to a complex interplay of regulatory events. In fact, the APC/C functions during mitosis and $G_{1}$ while it is kept inactive during S-phase (Figure 2). Analogous to Mus81 regulation, cyclin B-CDK and Cdc5-mediated phosphorylation contribute to APC/C activation during mitosis [52-54]. These events precede association of the APC/C with one of its co-activators, Cdc20, which functions as a substrate receptor and stimulates the catalytic activity of the ligase. APC/C ${ }^{\mathrm{Cdc} 20}$ subsequently targets securin and cyclin B for degradation, in a process that is tightly controlled by the spindle assembly checkpoint (SAC) [47]. This mode of regulation is strikingly reminiscent of the CDK- and Cdc5-mediated activation of Mus81 nuclease at the $\mathrm{G}_{2} / \mathrm{M}$ transition, 
which similarly promotes disjoining of chromosome-chromosome linkages (Figure 2).

Anaphase and mitotic exit are characterized by a decline in CDK activity that is accompanied by activation of cell cycle phosphatases such as Cdc14. This leads to the replacement of Cdc20 by Cdh1, a co-activator of the APC/C that is negatively regulated by CDK [46]. This switch is mirrored by the inactivation of Mus81 at anaphase and concomitant activation of Yen1 through declining CDK-mediated inhibition, as well as actively promoted dephosphorylation by Cdc14 $[33,38]$. While Yen1 ensures the elimination of any remaining recombination intermediates, APC/C ${ }^{\mathrm{Cdh} 1}$ targets - among others - Cdc20 itself leading to exit from mitosis. Yen1 and $A P C / C^{C d h 1}$ remain active in $G_{1}$ until entry into $S$ phase, at which stage S-phase CDK inhibits both enzymes. The APC/C ${ }^{\text {Chn } 1}$ complex ensures that cyclins are kept at low levels providing the cell with sufficient time before engaging in another cell division cycle; the role of active, nuclear Yen1 in $\mathrm{G}_{1}$ is currently unknown, yet it may be required to resolve repair intermediates deriving from spontaneous DNA lesions.

The overall emerging picture is that Mus81, Yen1, $\mathrm{APC} / \mathrm{C}^{\mathrm{Cdc} 20}$ and $\mathrm{APC} / \mathrm{C}^{\mathrm{Cdh} 1}$ activities are dampened during S-phase. This allows cells to accumulate securin and establish cohesin-based chromosomal connections and prevents Mus81 and Yen1 from prematurely resolving DNA-based chromosomal connections. Hence, cells appear to utilize a common strategy to coordinate the establishment and disengagement of protein-based and DNA-based chromosomal connections with cell cycle progression and cell division. 


\section{Conclusions and outlook}

Cell cycle regulation of Mus81-Mms4 and Yen1 appears to serve the simple need of yeast cells to moderate JM resolution specifically during S-phase. This prevents competition with STR, which processes JMs without the danger of eliciting reciprocal genetic exchanges (COs). At the same time, it also ensures that structures that are refractory to STR, such as single HJs or nicked HJs are processed in time for chromosome segregation and cell division (Figure 1).

Despite the recent advances, many questions concerning the regulation of JM processing remain open. We will only enumerate a few of them: 1) is the restraint of Mus81-Mms4 and Yen1 activities during S-phase important to prevent toxic processing of vital DNA replication and repair intermediates? Since both nucleases are capable of processing a variety of branched DNA structures in vitro, their late activation may contribute to genome stability in yet unanticipated ways; 2) how does phosphorylation regulate Mus81-Mms4 and Yen1 nucleases at the molecular level? While some insight has already been obtained for Yen1, it remains unclear how phosphorylation of Mms4 may enhance the activity of Mus81. To fully answer this question, a structural biology approach will be required. Another interesting aspect of Mus81-Mms4 phosphorylation is that it also regulates interaction with the scaffold proteins Dpb11/TOPBP1 and Slx4, which coordinate the cellular response to replication fork stalling $[36,55]$. How phosphorylation regulates the formation of the Mus81-Mms4-Dpb11-SIx4 complex is an interesting research topic; it remains to be investigated whether in addition to phosphorylation also other posttranslational modifications, such as sumoylation, may play a role in 
regulating Mus81-Mms4 function [56,57]; 3) is Mus81-Mms4 and Yen1 regulation important during meiosis? While initial work demonstrated that both enzymes have important roles and are tightly regulated during meiosis, it remains unclear whether regulation of their activities is important for successful haploidization; 4) do other organisms control Mus81 and Yen1/GEN1 activities? How? While initial work implies that fission yeast as well as human cells tightly regulate Mus81 and Yen1/GEN1 (absent in fission yeast) $[20,35]$, the precise mechanisms remain poorly understood. Furthermore, important functions for Mus81 and GEN1 orthologs have been identified in a variety of other organism, including worms and flies [58,59]. It will be interesting to understand whether such organisms employ similar strategies to yeast in establishing a hierarchy in pathway usage.

In summary, while much insight has been gained in recent years, how and why cells confine the activity of JM processing enzymes to defined temporal windows is only starting to be elucidated. Future work holds the promise of uncovering an even more elaborate regulatory network with important functions for maintenance of genome stability.

\section{References and recommended reading}

Papers of particular interest, published within the period of review, have been highlighted as:

- of special interest

•• of outstanding interest 


\section{Acknowledgements}

We thank members of the Matos lab, Fabienne Lampert, Ana-Maria Farcas and Vikram Panse for critical reading of the manuscript. The Matos laboratory is funded by ETH Zürich and the Swiss National Science Foundation (SNSF). Philipp Wild is supported by an EMBO long-term fellowship (ALTF 475-2015), co-funded by Marie Curie Actions of the European Commission (LTFCOFUND2013, GA-2013-609409).

\section{References}

1. Sancar A, Lindsey-Boltz LA, Unsal-Kacmaz K, Linn S: Molecular mechanisms of mammalian DNA repair and the DNA damage checkpoints. Annu Rev Biochem 2004, 73:39-85.

2. Lindahl T, Barnes DE: Repair of endogenous DNA damage. Cold Spring Harb Symp Quant Biol 2000, 65:127-133.

3. Heyer WD: Regulation of recombination and genomic maintenance. Cold Spring Harb Perspect Biol 2015, 7:a016501.

4. Symington LS, Gautier J: Double-strand break end resection and repair pathway choice. Annu Rev Genet 2011, 45:247-271.

5. Ira G, Malkova A, Liberi G, Foiani M, Haber JE: Srs2 and Sgs1-Top3 suppress crossovers during double-strand break repair in yeast. Cell 2003, 115:401-411.

6. Prakash R, Satory D, Dray E, Papusha A, Scheller J, Kramer W, Krejci L, Klein H, Haber JE, Sung P, et al.: Yeast Mph1 helicase dissociates Rad51made D-loops: implications for crossover control in mitotic recombination. Genes Dev 2009, 23:67-79.

7. Barber LJ, Youds JL, Ward JD, Mcllwraith MJ, O'Neil NJ, Petalcorin MI, Martin JS, Collis SJ, Cantor SB, Auclair M, et al.: RTEL1 maintains genomic 
stability by suppressing homologous recombination. Cell 2008, 135:261271.

8. Holliday R: A mechanism for gene conversion in fungi. Genet Res 2007, 89:285-307.

9. Liu Y, West SC: Happy Hollidays: 40th anniversary of the Holliday junction. Nat Rev Mol Cell Biol 2004, 5:937-944.

10. Neelsen KJ, Lopes M: Replication fork reversal in eukaryotes: from dead end to dynamic response. Nat Rev Mol Cell Biol 2015, 16:207-220.

11. Wu L, Hickson ID: The Bloom's syndrome helicase suppresses crossing over during homologous recombination. Nature 2003, 426:870874.

12. Ip SC, Rass U, Blanco MG, Flynn HR, Skehel JM, West SC: Identification of Holliday junction resolvases from humans and yeast. Nature 2008, 456:357-361.

13. Cejka P, Plank JL, Dombrowski CC, Kowalczykowski SC: Decatenation of DNA by the S. cerevisiae Sgs1-Top3-Rmi1 and RPA complex: a mechanism for disentangling chromosomes. Mol Cell 2012, 47:886-896.

14. Boddy MN, Gaillard PH, McDonald WH, Shanahan P, Yates JR, 3rd, Russell P: Mus81-Eme1 are essential components of a Holliday junction resolvase. Cell 2001, 107:537-548.

15. Chen XB, Melchionna R, Denis CM, Gaillard PH, Blasina A, Van de Weyer I, Boddy MN, Russell P, Vialard J, McGowan CH: Human Mus81associated endonuclease cleaves Holliday junctions in vitro. Mol Cell 2001, 8:1117-1127.

16. West SC, Blanco MG, Chan YW, Matos J, Sarbajna S, Wyatt HD: Resolution of Recombination Intermediates: Mechanisms and Regulation. Cold Spring Harb Symp Quant Biol 2015.

17. Garcia-Luis J, Machin F: Mus81-Mms4 and Yen1 resolve a novel anaphase bridge formed by noncanonical Holliday junctions. Nat Commun 2014, 5:5652.

18. Schwartz EK, Heyer WD: Processing of joint molecule intermediates by structure-selective endonucleases during homologous recombination in eukaryotes. Chromosoma 2011, 120:109-127. 
19. Schwartz EK, Wright WD, Ehmsen KT, Evans JE, Stahlberg H, Heyer WD: Mus81-Mms4 functions as a single heterodimer to cleave nicked intermediates in recombinational DNA repair. Mol Cell Biol 2012, 32:30653080.

20. Wyatt HD, Sarbajna S, Matos J, West SC: Coordinated actions of SLX1SLX4 and MUS81-EME1 for Holliday junction resolution in human cells. Mol Cell 2013, 52:234-247.

21. Castor D, Nair N, Declais AC, Lachaud C, Toth R, Macartney TJ, Lilley

DM, Arthur JS, Rouse J: Cooperative Control of Holliday Junction Resolution and DNA Repair by the SLX1 and MUS81-EME1 Nucleases. Mol Cell 2013, 52:221-233.

22. Munoz IM, Hain K, Declais AC, Gardiner M, Toh GW, Sanchez-Pulido L, Heuckmann JM, Toth R, Macartney T, Eppink B, et al.: Coordination of structure-specific nucleases by human SLX4/BTBD12 is required for DNA repair. Mol Cell 2009, 35:116-127.

23. Svendsen JM, Smogorzewska A, Sowa ME, O'Connell BC, Gygi SP, Elledge SJ, Harper JW: Mammalian BTBD12/SLX4 assembles a Holliday junction resolvase and is required for DNA repair. Cell 2009, 138:63-77.

24. Fekairi S, Scaglione S, Chahwan C, Taylor ER, Tissier A, Coulon S, Dong $M Q$, Ruse C, Yates JR, 3rd, Russell P, et al.: Human SLX4 is a Holliday junction resolvase subunit that binds multiple DNA repair/recombination endonucleases. Cell 2009, 138:78-89.

25. Andersen SL, Bergstralh DT, Kohl KP, LaRocque JR, Moore CB, Sekelsky $\mathrm{J}$ : Drosophila MUS312 and the vertebrate ortholog BTBD12 interact with DNA structure-specific endonucleases in DNA repair and recombination. $\mathrm{Mol}$ Cell 2009, 35:128-135.

26. Dayani $Y$, Simchen G, Lichten M: Meiotic recombination intermediates are resolved with minimal crossover formation during return-to-growth, an analogue of the mitotic cell cycle. PLoS Genet 2011, 7:e1002083.

26 - This study shows that STR-mediated dHJ dissolution occurs early during the cell cycle and leads to formation of non-crossovers. In the absence of STR, dHJs are processed at later stages and lead to formation of both crossovers and non-crossovers.

27. Wechsler T, Newman S, West SC: Aberrant chromosome morphology in human cells defective for Holliday junction resolution. Nature 2011, 471:642-646. 
28. Blanco MG, Matos J, Rass U, Ip SC, West SC: Functional overlap between the structure-specific nucleases Yen1 and Mus81-Mms4 for DNA-damage repair in S. cerevisiae. DNA Repair (Amst) 2010, 9:394-402.

29. Ho CK, Mazon G, Lam AF, Symington LS: Mus81 and Yen1 promote reciprocal exchange during mitotic recombination to maintain genome integrity in budding yeast. Mol Cell 2010, 40:988-1000.

30. Hunter N: Meiotic Recombination: The Essence of Heredity. Cold Spring Harb Perspect Biol 2015, 7.

31. Zakharyevich K, Tang S, Ma Y, Hunter N: Delineation of joint molecule resolution pathways in meiosis identifies a crossover-specific resolvase. Cell 2012, 149:334-347.

32. Mullen JR, Kaliraman V, Ibrahim SS, Brill SJ: Requirement for three novel protein complexes in the absence of the Sgs1 DNA helicase in Saccharomyces cerevisiae. Genetics 2001, 157:103-118.

33. Blanco MG, Matos J, West SC: Dual control of Yen1 nuclease activity and cellular localization by Cdk and Cdc14 prevents genome instability. Mol Cell 2014, 54:94-106.

33 •- This study provides molecular insight to the spatiotemporal control of Yen1 activity by CDK and Cdc14. It shows that Yen1 activity during Sphase is detrimental in the presence of exogenously introduced DNA damage and induces formation of crossovers.

34. Matos J, Blanco MG, West SC: Cell-cycle kinases coordinate the resolution of recombination intermediates with chromosome segregation. Cell Rep 2013, 4:76-86.

34 This work demonstrates that misregulated expression of Cdc5 causes premature Mus81-Mms4 activation during S-phase, which leads to increased formation of crossover recombinants.

35. Dehe PM, Coulon S, Scaglione S, Shanahan P, Takedachi A, Wohlschlegel JA, Yates JR, 3rd, Llorente B, Russell P, Gaillard PH: Regulation of Mus81-Eme1 Holliday junction resolvase in response to DNA damage. Nat Struct Mol Biol 2013, 20:598-603. 
36. Gritenaite D, Princz LN, Szakal B, Bantele SC, Wendeler L, Schilbach S, Habermann $\mathrm{BH}$, Matos J, Lisby M, Branzei D, et al.: A cell cycle-regulated

SIx4-Dpb11 complex promotes the resolution of DNA repair intermediates linked to stalled replication. Genes Dev 2014, 28:16041619.

36 - This work shows that cycle-regulated SIx4-Dpb11 complex formation promotes Mus81-mediated resolution of DNA repair intermediates arising from stalled replication.

37. Gallo-Fernandez M, Saugar I, Ortiz-Bazan MA, Vazquez MV, Tercero JA: Cell cycle-dependent regulation of the nuclease activity of Mus81Eme1/Mms4. Nucleic Acids Res 2012, 40:8325-8335.

37 - This study reveals that Mus81 activity is regulated during the cell cycle.

38. Eissler CL, Mazon G, Powers BL, Savinov SN, Symington LS, Hall MC: The Cdk/Cdc14 module controls activation of the Yen1 holliday junction resolvase to promote genome stability. Mol Cell 2014, 54:80-93.

38 -• This study provides molecular insight to the spatiotemporal control of Yen1 activity by CDK and Cdc14. It shows that Yen1 activity during Sphase induces formation of crossovers.

39. Szakal B, Branzei D: Premature Cdk1/Cdc5/Mus81 pathway activation induces aberrant replication and deleterious crossover. EMBO J 2013, 32:1155-1167.

39 •. This study shows that Mus81 function is regulated during the cell cycle. Furthermore, it demonstrates that expression of constitutively active Mus81-Mms4 leads to aberrant DNA replication and increased formation of crossover recombinants.

40. Blanco MG, Matos J: Hold your horSSEs: controlling structureselective endonucleases MUS81 and Yen1/GEN1. Front Genet 2015, 6:253. 
41. Matos J, West SC: Holliday junction resolution: regulation in space and time. DNA Repair (Amst) 2014, 19:176-181.

42. Matos J, Blanco MG, Maslen S, Skehel JM, West SC: Regulatory control of the resolution of DNA recombination intermediates during meiosis and mitosis. Cell 2011, 147:158-172.

$42 \cdot$ A combination of genetic, biochemical and cell biology methods uncovers a regulatory network that connects cell cycle stage-specific phosphorylation events to JM processing by Mus81-Mms4 and Yen1 in meiosis and mitosis.

43. Hochegger $\mathrm{H}$, Takeda $\mathrm{S}$, Hunt $\mathrm{T}$ : Cyclin-dependent kinases and cellcycle transitions: does one fit all? Nat Rev Mol Cell Biol 2008, 9:910-916.

44. Archambault V, Glover DM: Polo-like kinases: conservation and divergence in their functions and regulation. Nat Rev Mol Cell Biol 2009, 10:265-275.

45. Kosugi S, Hasebe M, Tomita M, Yanagawa H: Systematic identification of cell cycle-dependent yeast nucleocytoplasmic shuttling proteins by prediction of composite motifs. Proc Natl Acad Sci U S A 2009, 106:1017110176.

46. Zachariae W, Nasmyth $\mathrm{K}$ : Whose end is destruction: cell division and the anaphase-promoting complex. Genes Dev 1999, 13:2039-2058.

47. Sivakumar S, Gorbsky GJ: Spatiotemporal regulation of the anaphasepromoting complex in mitosis. Nat Rev Mol Cell Biol 2015, 16:82-94.

48. Uhlmann F, Lottspeich F, Nasmyth K: Sister-chromatid separation at anaphase onset is promoted by cleavage of the cohesin subunit Scc1. Nature 1999, 400:37-42.

49. Sudakin V, Ganoth D, Dahan A, Heller H, Hershko J, Luca FC, Ruderman JV, Hershko A: The cyclosome, a large complex containing cyclinselective ubiquitin ligase activity, targets cyclins for destruction at the end of mitosis. Mol Biol Cell 1995, 6:185-197.

50. King RW, Peters JM, Tugendreich S, Rolfe M, Hieter P, Kirschner MW: A $20 S$ complex containing $C D C 27$ and $C D C 16$ catalyzes the mitosisspecific conjugation of ubiquitin to cyclin B. Cell 1995, 81:279-288. 
51. Irniger S, Piatti S, Michaelis C, Nasmyth K: Genes involved in sister chromatid separation are needed for B-type cyclin proteolysis in budding yeast. Cell 1995, 81:269-278.

52. Kraft C, Herzog F, Gieffers C, Mechtler K, Hagting A, Pines J, Peters JM: Mitotic regulation of the human anaphase-promoting complex by phosphorylation. EMBO J 2003, 22:6598-6609.

53. Charles JF, Jaspersen SL, Tinker-Kulberg RL, Hwang L, Szidon A, Morgan DO: The Polo-related kinase Cdc5 activates and is destroyed by the mitotic cyclin destruction machinery in S. cerevisiae. Curr Biol 1998, 8:497-507.

54. Shirayama M, Zachariae W, Ciosk R, Nasmyth K: The Polo-like kinase Cdc5p and the WD-repeat protein Cdc20p/fizzy are regulators and substrates of the anaphase promoting complex in Saccharomyces cerevisiae. EMBO J 1998, 17:1336-1349.

55. Princz LN, Gritenaite D, Pfander B: The SIx4-Dpb11 scaffold complex: coordinating the response to replication fork stalling in S-phase and the subsequent mitosis. Cell Cycle 2015, 14:488-494.

56. Reynolds A, Qiao H, Yang Y, Chen JK, Jackson N, Biswas K, Holloway JK, Baudat F, de Massy B, Wang J, et al.: RNF212 is a dosage-sensitive regulator of crossing-over during mammalian meiosis. Nat Genet 2013, 45:269-278.

57. Guervilly JH, Takedachi A, Naim V, Scaglione S, Chawhan C, Lovera Y, Despras E, Kuraoka I, Kannouche P, Rosselli F, et al.: The SLX4 complex is a SUMO E3 ligase that impacts on replication stress outcome and genome stability. Mol Cell 2015, 57:123-137.

58. Bailly AP, Freeman A, Hall J, Declais AC, Alpi A, Lilley DM, Ahmed S, Gartner A: The Caenorhabditis elegans homolog of Gen1/Yen1 resolvases links DNA damage signaling to DNA double-strand break repair. PLoS Genet 2010, 6:e1001025.

59. Andersen SL, Kuo HK, Savukoski D, Brodsky MH, Sekelsky J: Three structure-selective endonucleases are essential in the absence of BLM helicase in Drosophila. PLoS Genet 2011, 7:e1002315. 


\section{Figure legends}

Figure 1 - Processing of DNA joint molecules (JMs) during the cell cycle. Repair of unscheduled chromosomal lesions by homologous recombination leads to formation of DNA-based chromosomal connections between sister chromatids and, occasionally, homologous chromosomes. (A) During S-phase and G2, anti-recombinogenic helicases disengage the majority of early JMs by destabilizing displacement loop (D-loop) structures. Structures that mature to form double Holliday junctions (dHJs) are "dissolved" by the STR/BTR complex, to generate non-crossover (NCO) recombinants. Up-regulation of Mus81-Mms4/EME1 and Yen1/GEN1 nuclease activities during mitosis ensures the "resolution" of persistent JMs that escape the STR/BTR complex. These include dHJs but also single HJs and nicked HJs. Processing of late JMs by Mus81-Mms4/EME1 and Yen1/GEN1 can lead to formation of crossovers (COs) and drive loss of heterozygosity (LOH). (B) Depiction of key DNA JM intermediate structures that link recombining sister chromatids or homologous chromosomes. For easier visualization only inter-homolog JMs (blue-grey) are shown. (C) Sketch of a single Holliday junction connecting two DNA duplexes. (D) Illustration of the outcome of STR/BTR-dependent 'dissolution' and Mus81-Mms4/EME1- and Yen1/GEN1-dependent 'resolution' pathways. Convergent branch migration of two HJs catalyzed by the Sgs1/BLM helicase and subsequent Top3-dependent dissolution of the hemicatenane results in the formation of NCO recombinants. Conversely, nucleoytic resolution by Mus81-Mms4/EME1 or Yen1/GEN1 yields NCOs and COs with equal probability. Cleavage of the crossing strands (black arrows) 
prevents formation of COs. Processing of the non-crossing strands (red arrows) promotes formation of COs. The depicted NCO arises from cleavage of the crossing strands in both HJs. Cleavage of the non-crossing strands in one of the HJs (left) and cleavage of the crossing strands in the other $\mathrm{HJ}$ gives rise to the portrayed $\mathrm{CO}$. For simplicity, genetic exchange without $\mathrm{CO}$ formation is not shown.

Figure 2 - Activity profiles of S. cerevisiae Mus81-Mms4 and Yen1 nucleases and $\mathrm{APC} / \mathrm{C}$ ubiquitin ligase during the cell cycle. The activities of Mus81Mms4 and Yen1 are regulated by cell cycle stage-specific phosphorylation events. Mus81-Mms4, which is partly active during G1 and S-phase/G2, becomes fully activated at the G2/M transition, upon M-CDK- and Cdc5mediated phosphorylation. Yen1 is kept in an inactive state by S-CDKmediated phosphorylation until metaphase. Cyclin degradation and release of Cdc14 phosphatase from the nucleolus promotes rapid Yen1 dephosphorylation and its concomitant activation at anaphase. Yen1 remains active until the G1/S-phase transition of the subsequent cell cycle.

Analogous to Mus81-Mms4 and Yen1 regulation, the APC/C complex is positively and negatively regulated by CDK-dependent phosphorylation events. M-phase CDK and Cdc5 promote APC/C activation at the G2/M transition. APC/C activation also requires association to Cdc20, which is negatively regulated by the spindle assembly checkpoint (SAC) (orange dashed line depicts phosphorylated APC/C that is prevented from interacting with $\mathrm{Cdc} 20$ by $\mathrm{SAC})$. APC/C $\mathrm{C}^{\mathrm{Cdc} 20}$ triggers the metaphase-anaphase transition. Decreasing cyclin levels and Cdc14 activation lead to replacement of Cdc20 
by Cdh1, which maintains the activity of APC/C high until S-CDK activity rises at the $\mathrm{G} 1 / \mathrm{S}$ transition of the following cell cycle. 
A

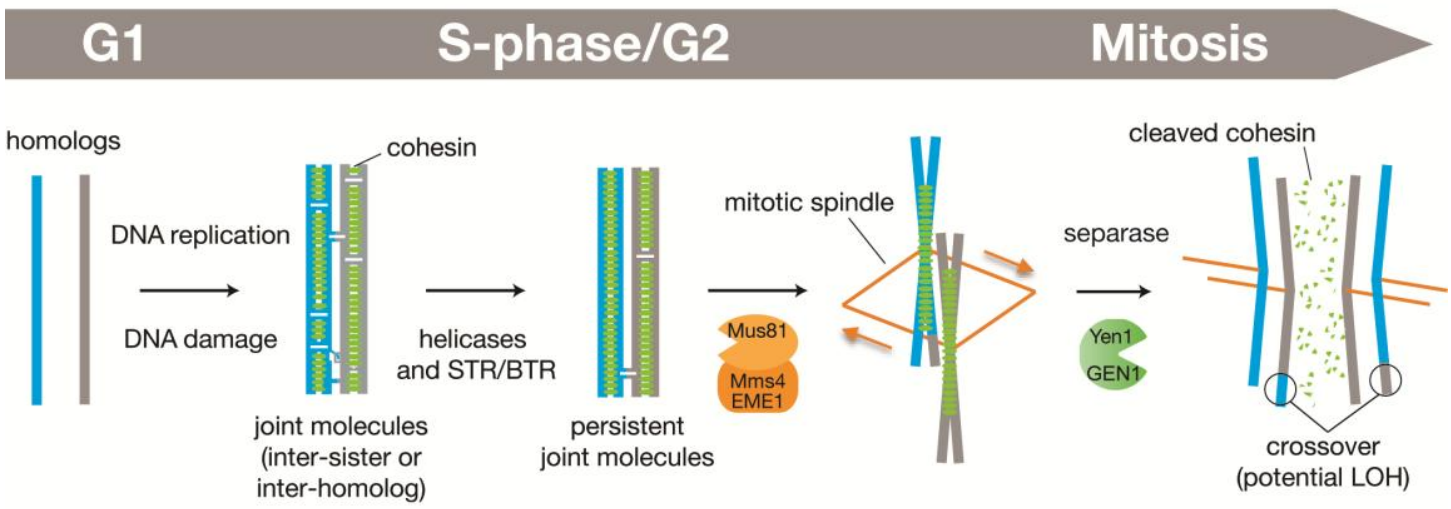

B

D-loop

C

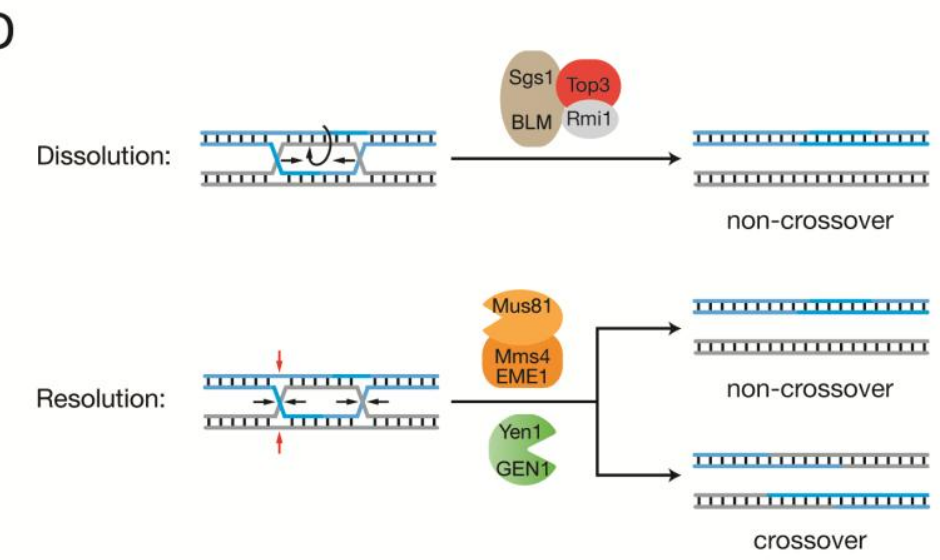

Wild and Matos, Figure 1 


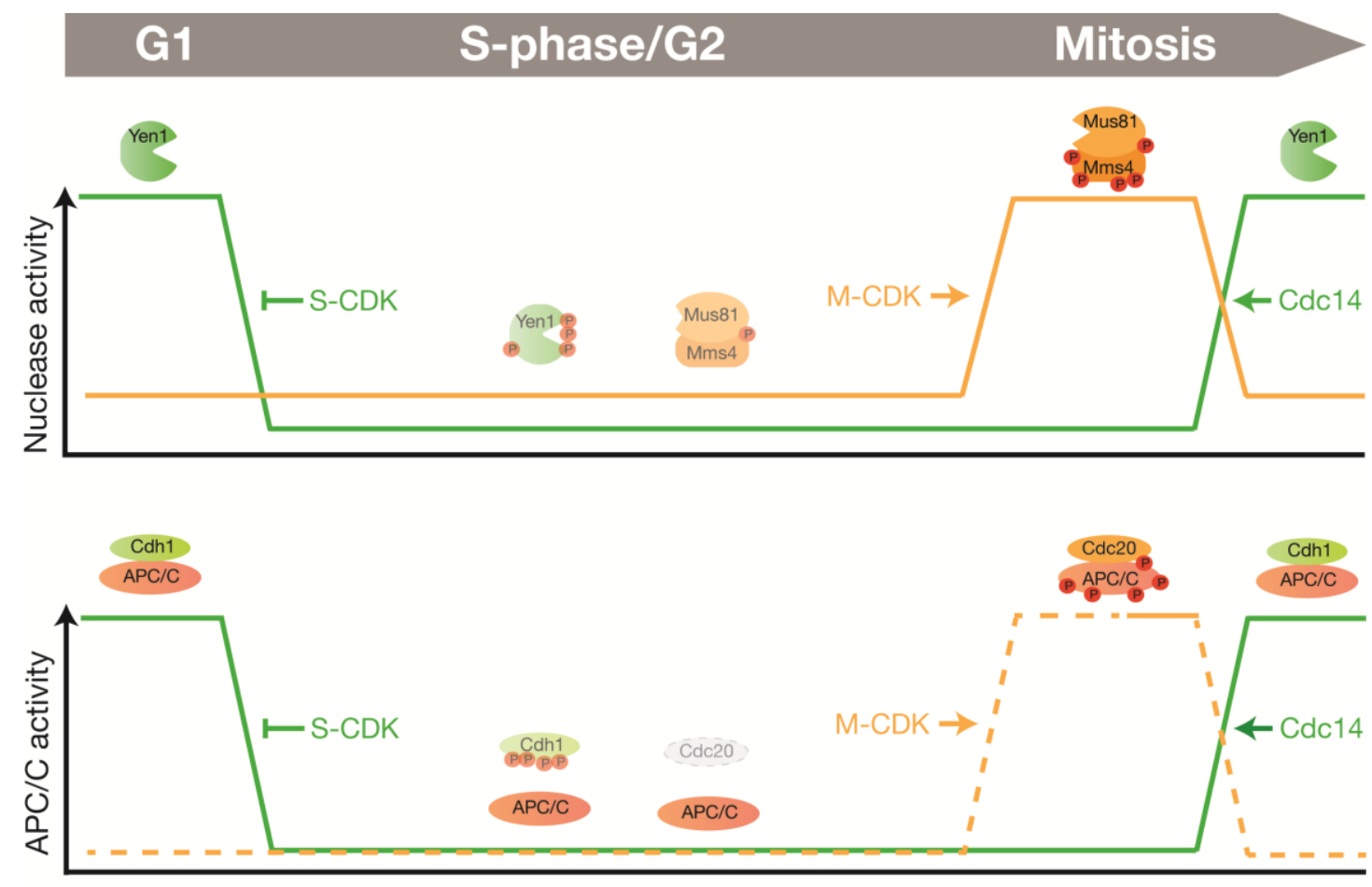

Wild and Matos, Figure 2 